\title{
Mathematical model of consolidation of fine concrete mixtures with different mobility, casted by vacuumizing and axial pressing in layers
}

\author{
Elena Dedeneva ${ }^{1,}$, Ibrahim Kazimagomedov ${ }^{1}$, Saad Salem ${ }^{1}$, Tatiana Kostyuk ${ }^{1}$, \\ Younis Basheer N. ${ }^{1}$, and Marina Yakimenko ${ }^{1}$ \\ ${ }^{1}$ Kharkiv National University of Civil Engineering and Architecture, 61002 Kharkiv, Ukraine
}

\begin{abstract}
A mathematical model allowing establishing regularities in the consolidation processes of fine-grained concrete mixtures with different mobility and compaction methods has been worked out. This study is based on two-phase systems and nonlinear character of their consolidation. It resolves the question of the choice of vacuumizing optimal parameters and axial pressing in layers for molding of thin-walled products such as concrete roof tiles and concrete pipe products. Finally, we can get products without heat treatment by the materials and energy-saving technologies.
\end{abstract}

\section{Relevance of the work}

Development and implementation of a new generation of concrete are considered very important for modern construction that will allow realizing the most interesting architectural projects. Concrete production is based on a thorough study of the processes that reveals new patterns of structure at different levels: micro-, meso- and macro-levels. The concrete structure is the main determining factor of its basic physical and mechanical properties. Therefore, the study of methods of forming a homogeneous structure, achieving high levels of strength at early stages of hardening, and producing highly functional concrete is extremely important. Dependencies between concrete structure and its properties are common regularities for all kinds of concrete, which consider the structural components of the composite theory. By installing these regularities, we can impact concrete structure, form its properties in order to obtain concretes of specified technical parameters $[1,2,3]$. Modern technology intensive construction methods require removing mold forming in short time having a sufficient quality of concrete. Therefore, High Performance Concrete (HPC) characterized by high operating ability is more frequently used [3, 4]. The main branches of the HPC use are the high-rise construction, nuclear power plants, waterworks, pipelines, bridges and engineering structures, road surfaces of monolithic and precast-monolithic structures, runways of airfields, launching complexes for space systems and other special units [2].

Recently, as a result of sharp increase in intensity of traffic flows, environmental degradation and impact of aggressive environment, all these factors have led to increasing

\footnotetext{
*Corresponding author: kstuca.bmiv@,gmail.com
} 
the load on reinforced concrete structures of transport objects, bridges, overpasses, tunnels, and deformation of these structures, thus requiring constant repair. It is known [3] that after a long operation local defects in the concrete cover appear in the elements of reinforced concrete bridges. It assists to protect the steel bars. The whole evolution of the improvement of the concrete structure to increase its strength is associated with a decrease in the size of coarse aggregate from 20-40 to 3-10 $\mathrm{mm}$ [4]. Thus, fine-grained concrete is increasingly in demand. Fine-grained concrete is used for production of load-bearing elements of concrete and reinforced concrete structures. As it is known, the structure of concrete is the main and determining factor of its physical-mechanical properties. It is compacted both during the molding and curing processes. The period of formation of the concrete structure and its properties depend on the composition of the initial materials and the method of molding [4 - 7].

For the selection of optimal parameters of effective methods for compacting concrete and mortar mixtures, a mathematical model with the establishment of appropriate regularities in the course of these complex processes is important for theoretical justification.

The object of the authors' research is thin-walled products in the form of concrete pipes (circular and trough configuration) and flat concrete tiles. In Ukraine, as well as in the world, there is a great demand for roofing materials and materials for water disposal and land reclamation. Concrete materials of this functional purpose allow replacing expensive ceramic tiles and pipes, as well as environmentally unsafe asbestos-cement materials.

By analogy with the study of consolidation processes in soils, this paper is based on the biphasic of concrete systems: solid and gaseous one in rigid mixtures and solid and liquid one in ductile [3]. At the first stage, consolidation, the particles of the "skeleton" are mechanically approached and the material acquires new properties, in particular the structural strength $R_{s t r}$ without chemical transformations of the clinker minerals that make up the molding mixture. At the hardening stage, active crystallization processes take place, in the result of which the material of building products acquires specified properties (strength, density, etc.). The problems of accelerating the molding process, both at the first and second stages, have great importance for the theory and practice of obtaining building materials and products.

The solution of the nonlinear equation of consolidation allows us to link process parameters, load, time of its application, thickness of the layer of the material compacted, with the physical and mechanical characteristics of the molding mixture obtained on the basis of an experimental study of samples of the mixture at a compression test. The processing of results allows us to establish the dependence of the relative porosity $\psi$ on the effective pressure $\sigma_{e f f}$, the filtration coefficient $K_{f}$ on the relative porosity, and on the basis of the obtained data to calculate the constants for each mixture composition. It is essential that the description of the consolidation process of molding mixtures is carried out within the framework of a mathematical model for mixtures of various mobility both tough and low-plastic ones.

\section{Research and discussion of the results}

The process of molding mixture compaction is similar to soil compaction in the approach of the particles of the composite (crushed stone, sand, cement and bound water) with filtration of the medium filling the gaps in the skeleton. If the mixture is rigid, is air (axial pressing by layers) if it is plastic (vacuumizing) the filtered medium is the water-cement mixture. In the end of the consolidation process, when the particles will be closer to the contact of the bound water shells (the end of the filtration process) the material acquires new properties and, most importantly, structural strength, which allows removing mold forms immediately. 
Further increase in the applied load (vacuum) does not lead to changes in mechanical properties, since even a high-pressure fluid application cannot delete associated fails. The consolidation process is very fleeting, thus, the more rigid moldable mixture, the faster it compacts Thus, in the case of axial pressing in layers, the compaction of a layer up to onecentimeter-thick takes less than a second, and in the case of vacuumizing, it takes 5-7 seconds. This makes it possible to disregard processes of dissolution and hydrolysis of the initial particles of the binder and, especially, crystallization of calcium hydro silicate and calcium hydroxide in the pore electrolyte, but the binder is water, and there are processes of dissolution and hydrolysis of the initial, especially small particles of the binder, and formation of contacts between original particles and ones of a new formation [5]. The material constants can be determines as follows

$$
\xi=\frac{y h}{\sqrt{2 \theta_{i} C_{k} t}}
$$

where $h$ is the thickness of a compacted layer; $C_{k}$ is the coefficient of consolidation in compression; $t$ is the loading duration; $\theta=c e^{-0.5(T-1) q} ; q$ is the compression pressure; $a, n, \alpha$ are dimensionless parameters determined by compression testing and incoming depending on

$$
\begin{aligned}
& \psi=c e^{-\alpha q} \\
& K_{f}=K_{f}^{0} \psi^{n}
\end{aligned}
$$

Relating the degree of compaction of the concrete mix with the process parameters compression tests of fine mixtures were held at different values of $\mathrm{w} / \mathrm{c}$ and humidity of a mixture as shown in Table 1 below. There are specified values of structural strength $R_{\text {str }}$ determined experimentally.

Table 1. The dependence of structural strength of concrete mixtures on moisture and water-cement ratio.

\begin{tabular}{|c|c|c|c|}
\hline $\begin{array}{c}\text { Number of } \\
\text { composition }\end{array}$ & $\begin{array}{c}\text { Water-cement } \\
\text { ratio, W/C }\end{array}$ & $\begin{array}{c}\text { Concrete } \\
\text { moisture, W, } \%\end{array}$ & $\begin{array}{c}\text { Structural strength } R_{\text {str }}, \\
\mathrm{kg} / \mathrm{cm}^{2}\end{array}$ \\
\hline 1 & 0.33 & 5.25 & 0.42 \\
\hline 2 & 0.42 & 5.25 & 0.41 \\
\hline 3 & 0.42 & 6.8 & 0.31 \\
\hline 4 & 0.39 & 18.0 & 0.42 \\
\hline 5 & 0.41 & 18.9 & 0.40 \\
\hline
\end{tabular}

Compression tests were carried out in a cylinder of a diameter of $51 \mathrm{~mm}$ with a pressure from 0.1 to $0.5 \mathrm{MPa}$, stepwise, with a uniform step of $0.1 \mathrm{MPa}$, which completely covers the range of pressures used for axial pressing in layers. A vacuum mold form with dimensions of $20 \times 20 \times 5 \mathrm{~cm}$ was also used for tests of fine-grained mixtures compacted by vacuumizing. The range of generated vacuum pressures is from $0.02 \mathrm{MPa}$ to $0.1 \mathrm{MPa}$ with an interval of $0.02 \mathrm{MPa}$, which corresponds to the values of generated pressures in the molding of concrete roof tiles. As shown by compression studies of concrete mixtures in a wide range of composition and humidity changes [4], the coefficient of initial filtration $K_{f}^{o}$ values from $1.8 \times 10^{-7}$ to $7.4 \times 10^{-7}$. The graphical dependence of the relative porosity on the effective pressure for mixtures 1-5 is shown in Fig. 1 in semi logarithmic coordinates. 


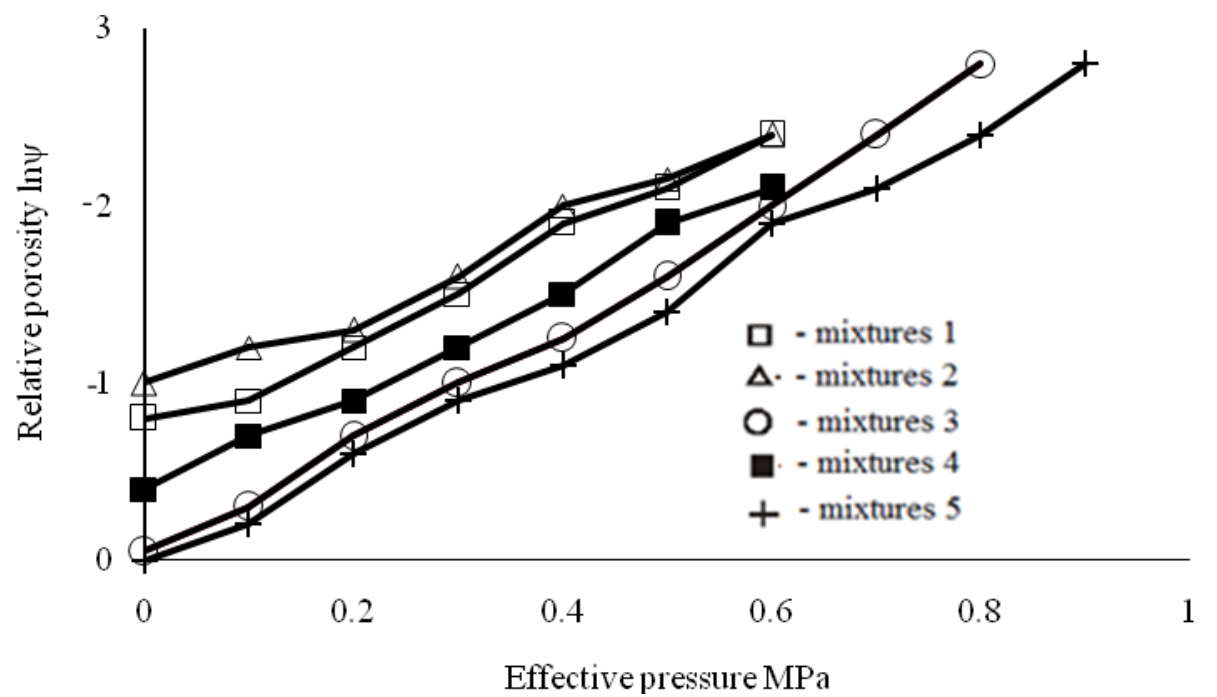

Fig. 1. Dependence of the relative porosity on the effective pressure.

As the filtration characteristics of concrete mixtures to the value $\ln \psi=-1.0$ showed, the $\ln \frac{K_{f}}{K_{f}^{o}}$ dependence is linear and the determination of the coefficient $n$ in expression (3) does not run into difficulties. So for the considered compositions, $n=1.75$ and the parameters of the compression curves $c=0.905-1.0, \alpha=3.5-3.33$, that is, with sufficient accuracy for practical application, can be set $\mathbf{c}=1.0, \alpha=3.4, n=1.75$.

Expression (1) includes all the basic technological parameters of the compaction process under axial compression by layers, and vacuumizing.

\section{Conclusions}

It has been established that, when molding concrete roof tiles, the application of the linear compaction theory leads to an increase of the vacuumizing time necessary to obtain a given structural strength. Thus, when the shingles are formed with a specified structural strength of $0.42 \mathrm{~kg} / \mathrm{cm}^{2}$ and a thickness of $12 \mathrm{~mm}$ at the vacuum pressure $0.05 \mathrm{MPa}$, the time required for the nonlinearity process is 9 seconds, and according to the linear theory it is 15 seconds. Thus, the nonlinear model for the consolidation processes of solution and finegrained mixtures, compacted by vacuuming and axial pressing in layers, proposed by the authors, makes it possible to obtain thin-walled products with a sufficiently high structural strength without thermal treatment in terms of material and energy-saving technologies.

\section{References}

1. R. Runova, V. Gotz., M. Sanitsky, Konstruktsiyni materiali new pokolinnya that tehnologii ih vprovadzhennya in budivnitstvo (2008)

2. Z. Jamrozy, Beton i jego technologie (Warzawa, Wydawnictwo naukowe Pwn, 2000)

3. N. Zaichenko, High-strength fine-grained concrete with a complex microstructure of the modified (2009). 
4. O. Vandolovsky, I. Kazimagomedov E., O. Dedenova, News ODABA 35, 50-55 (2009)

5. A.Vandolovsky, E. Dedeneva, HDTUBA HOTV OMB, 41, 127 (2007)

6. A. Vandolovsky, E. Dedeneva, HDTUBA HOTV OMB, 47, 192 (2008)

7. B. Younis, E. Dedeneva, T. Kostiuk, Modern scientific achievements: experience exchange, Morrisville, Lulu Press, 28-31 (2017) 